\title{
Nanoscale Imaging Mass Spectrometry using Cluster Ion Beams.
}

\author{
Nicholas Winograd
}

Department of Chemistry, Penn State University, University Park, PA 16875.

Imaging mass spectrometry has emerged, in recent years, to allow chemical composition to be elucidated with impressive spatial resolution and sensitivity. Many different approaches have been developed, each with specific attributes. Techniques employing soft ionization schemes such as MALDI and desorption electrospray ionization (DESI) have been successful particularly for biological samples since the target molecule is generally detected without fragmentation. Spatial resolution is generally limited to $100 \mu \mathrm{m}$, although better resolution has been reported in special cases. Here, we focus on molecular secondary ion mass spectrometry (SIMS) using focused cluster ion beams to stimulate the desorption of molecular ions [1]. Since desorption results from energetic collisions between the primary cluster and the target molecule, significant fragmentation of molecular ions is usually observed. Despite this problem, there are several unique operational modes of the SIMS approach that present a unique space for materials characterization. These modes include the ability to acquire surface-specific chemical information and to perform 3dimensional imaging with sub-micron spatial resolution and nanometer-scale depth resolution. This approach is distinct from nanoSIMS where the energetic ion beam intentionally fragments the molecules to small fragments which are distinguished using stable isotope labeling.

The development of cluster ion beams for imaging SIMS has been a real success story. About 15 years ago, it was shown that metal cluster ions such as $\mathrm{Au}_{3}$ and $\mathrm{Bi}_{3}$ could be generated from liquid metal ions sources and focused to a spot size of less than $100 \mathrm{~nm}$ [2]. These clusters were shown to produce mass spectra with much higher ion yields and less fragmentation than the more conventional atomic ion projectiles. Ion sources employing bigger clusters such as $\mathrm{C}_{60}$ were also commercialized and incorporated into existing time-of-flight (TOF)-SIMS machines [3]. These probes could not be focused as well as the liquid metal ion sources, but could still yield spatial resolutions in the range of $300 \mathrm{~nm}$. Most recently, gas cluster ion beams (GCIB) produced by supersonic expansion, have been perfected for SIMS imaging, yielding probes consisting of, for example, argon clusters ranging in size from several hundred to several thousand atoms [4].

A critically important ancillary benefit of the cluster ion sources is that there is minimal chemical damage accumulation during erosion of the sample. The information in the mass spectrum is retained as a function of depth into the sample. Many studies using $\mathrm{C}_{60}$, for example, on well-defined layered structures such as those containing Irganox polymer additives, and lipid bilayers, showed that 10-20 nm depth resolution could be achieved through a thickness of several microns. Moreover, the GCIB sources are now able to discern sub-surface layers with $5-10 \mathrm{~nm}$ resolution. Hence, it is possible to acquire images with submicron spatial resolution, and to stack these images during molecular depth profiling to achieve a 3dimensional chemically-resolved picture with nanometer depth resolution [5].

There are two major problems that need to be resolved before this approach can be routinely applied. First, and foremost, is the issue of sensitivity. As the spot size of the primary ion beam is reduced toward zero, the number of molecules available for detection also approaches zero. Moreover, ionization efficiency for any of these desorption methods is thought to be in the range of 1 molecule in $10^{8}$ to one molecule in $10^{4}$, a very rare event indeed. The ultimate spatial resolution is generally limited by sample 
size, rather than by how tightly the ion beam can be focused, especially consider there are only $\sim 10^{6}$ molecules per layer per square micron. There is a second conundrum associated with the GCIB sources. With smaller cluster projectiles, ions are produced in greater quantity, but more molecules are fragmented, reducing the ionization efficiency. With the larger clusters, molecular desorption is very efficient, but the "softness" of the ionization means that the ionization efficiency is poor. In this talk, various solutions to this conundrum will be explored, including the use of Argon clusters doped with $\mathrm{H}$-containing species to enhance $[\mathrm{M}+\mathrm{H}]^{+}$formation.

The unique properties associated with cluster SIMS have altered the instrumentation landscape. The low duty cycle associated with traditional TOF-SIMS instruments complicates depth profiling and image quality. There has been a recent move toward the use of continuous ion beams, rather than pulsed ion beams to circumvent these complications. Several options will be discussed in this talk, including the use of a shaped field buncher to time focus a section of the continuous beam, which is then accelerated into a harmonic reflectron [6].

Finally, we present several applications of 2 and 3-dimensional imaging using cluster beams to characterize a variety of biological samples. For these applications, sample preparation is a key part of the measurement. For example, we are able to show the lipid distribution at the single cell level for HeLa cells by combining the high spatial resolution of the $\mathrm{C}_{60}$ source with the improved molecular desorption properties of the GCIB. In addition, we show that to achieve the best spatial resolution using tissue slices, it is necessary to avoid using a matrix coating, and to perform the measurements at cryogenic temperatures to avoid molecule migration and evaporation. Other issues such as the presence of matrix ionization effects, differential sputtering and charging will be discussed in detail. In general, the lecture will attempt to provide an assessment of where the technique stands with respect to other imaging mass spectrometry methods, what the current challenges are for improvement, and to speculate about future developments in the coming years.

\section{References:}

[1] J. C. Vickerman and N. Winograd, International Journal of Mass Spectrometry, ASAP (2015). DOI: 10.1016/j.ijms.2014.06.021

[2] D. Touboul et al., J. Am. Soc. Mass Spectrom. 16 (2005), p. 1608-1618.

[3] D. Weibel et al., Anal. Chem. 75 (2003), p. 1754-1764.

[4] N. Toyoda et al., Appl. Surf. Sci. 203 (2003), p. 214-218.

[5] N. Winograd, Surf. Interface. Anal. 45 (2013), p. 3-8.

[6] R. Hill et al., Surf. Interface Anal. 43 (2011), p. 506-509.

[7] Funding from the Department of Energy (grant no. DE-FG02-06ER15803), The National Science

Foundation (grant no. CHE-0908226) and the National Institutes of Health (grant no 9R01 GM11374620A1) are gratefully acknowledged. 